\title{
EFEITO DE SISTEMAS DE PRODUÇÃO DE GRÃOS E DE PASTAGENS SOB PLANTIO DIRETO SOBRE O NÍVEL DE FERTILIDADE DO SOLO APÓS CINCO ANOS ${ }^{(1)}$
}

\author{
H. P. SANTOS ${ }^{(2)}$, R. S. FONTANE LI ${ }^{(3)} \&$ G. O. TOMM(4)
}

\begin{abstract}
RESUMO
A fertilidade do solo foi avaliada num Latossolo Vermelho distrófico típico, em Passo F undo (RS), após cinco anos de estabelecimento (1993 a 1997) de quatro sistemas de produção, integrando grãos, pastagens anuais de inverno e pastagens perenes: sistema I (trigo/soja, aveia branca/soja e ervilhaca/milho); sistema II (trigo/soja, aveia branca/soja e aveia preta + ervilhaca pastejadas/milho); sistema III [pastagens perenes da estação fria (festuca + trevo branco + cornichão)]; sistema IV [pastagens perenes da estação quente (pensacola + aveia preta + azevém + trevo vermelho + cornichão)], e sistema V (alfafa para feno), acrescentado como tratamento adicional com repetições em áreas contíguas ao experimento em 1994. Os tratamentos foram distribuídos em blocos ao acaso com quatro repetições. Os si stemas de produção, sob plantio di reto, elevaram os teores de matéria orgânica, de $\mathbf{P}$ extraível e de $\mathbf{K}$ trocável, principalmente na camada de solo de $0-5 \mathrm{~cm}$. Nos quatro primeiros sistemas de produção, houve aumento dos valores de pH e de $\mathrm{Ca}+\mathrm{Mg}$ trocáveis, da superfície $(0-5 \mathrm{~cm})$ para a camada mais profunda do solo $(15-20 \mathrm{~cm})$, enquanto com o valor de Al trocável ocorreu o contrário. Os sistemas com pastagens perenes mostraram maior teor de matéria orgânica na camada superficial do solo do que os sistemas com pastagens anuais.
\end{abstract}

Termos de indexação: rotação de culturas, integração lavoura-pecuária, pastagem anual e perene.

\footnotetext{
(1) Recebido para publicação em junho de 2000 e aprovado em março de 2001.

(2) Engenheiro-Agrônomo, Dr., E mbrapa-Centro Nacional de Pesquisa de Trigo (Embrapa Trigo). Caixa Postal 451, CEP $99001-970$ Passo Fundo (RS). Bolsista CNPq-PQ. E.mail: hpsantos@cnpt.embrapa.br

(3) Engenheiro-Agrônomo, Ph.D., Embrapa Trigo e Professor Titular da FAMV-UPF.

(4) Engenheiro-Agrônomo, Ph.D., Embrapa Trigo.
} 


\title{
SUMMARY: EFFECT OF GRAIN AND FORAGE CROP PRODUCTION SYSTEMS UNDER NO-TILLAGE ON SOIL FERTILITY AFTER FIVE YEARS
}

\begin{abstract}
Soil fertility characteristics wereeval uated on a typical dystrophic Red L atosol located in Passo F undo, Rio Grande do Sul, B razil , after fiveyears of combined production systems (1993 to 1997). The effects of integrated grain production systems and with winter annual and perennial pastures under no-tillage were assessed. Four production systems were evaluated: system I (wheat/ soybean, whiteoat/ soybean, and common vetch/ corn); system II (wheat/ soybean, white oat/ soybean, and grazed black oat + grazed common vetch/ corn); system III [perennial cool season pastures (fescue + white clover + birdsfoot trefoil)]; and system IV [perennial warm season pastures (bahiagrass + black oat + ryegrass + red clover + birdsfoot trefoil)]; and system $V$ (alfalfa for forage), which was established in an adjacent area in 1994. The treatments were arranged in a randomized complete block design, with four replications under no-tillage The producti on systems increased the contents of organic matter, extractableP, and exchangeabl eK, mainly at 0-5 cm depth. Therewas an increase in the $\mathrm{pH}$ value and exchangeable $\mathrm{Ca}+\mathrm{Mg}$ contents in thefirst four production systems from the soil surface $(0-5 \mathrm{~cm})$ to deeper layers $(15-20 \mathrm{~cm})$. The reverse occurred with values of exchangeableAl. Thesystems with grazed perennial pastures showed higher organic matter content at soil surface, as compared to systems with annual pastures.
\end{abstract}

Index terms: crop rotation, crop - pasture integration, annual and perennial pasture

\section{NTRODUÇÃO}

A integraçãolavoura-pecuária, sob plantio direto (PD), tem mostrado que étécnica eeconomicamente viável (Mello, 1996). Para tal, devem ser identificados sistemas de produção de média e longa duração, integrando a produção de grãos com a de pastagens perenes, os quais ofereçam maior sustentabilidade e melhor resultado econômico possível. Além disso, éimportanteincluir nas pastagens leguminosas que fixem $\mathrm{N}$ e melhorem o valor nutritivo da forragem, contribuindo para aumentar a produção animal e para mel horar as condições físico-químicas do solo. As leguminosas, sempre que possível, devem ser incluídas no planejamento de sistemas de produção ou de rotação de culturas.

Os sistemas de produção mistos que incluem a combinação de pastagens perenes, al ém de culturas anuais para produção degrãos, são os mais eficientes na manutenção da estrutura físico-química do solo favorável às plantas (Carpenedo \& Mielniczuk, 1990). As pastagens perenes exercem seus efeitos por períodos prolongados; as gramíneas apresentam sistema radicular extenso e em constanterenovação, e os resíduos das leguminosas contribuem com nitrogênio, levando ao aumento na taxa de decomposição dos materiais orgânicos por reduzir a relação C/N (Harris et al., 1966).

Do ponto de vista químico, em geral, os solos cultivados sob plantio direto apresentam maior concentração de $\mathrm{Ca}+\mathrm{Mg}$ trocáveis, de matéria orgânica, de $\mathrm{P}$ extraível e de K trocável na camada superficial do perfil $(0-5 \mathrm{~cm})$ (Shear \& Moschler,
1969; TriplettJ r. \& Van Doren J r., 1969; M uzilli, 1983; Sidiras \& Pavan, 1985; Sá, 1993; Santos \& Tomm, 1996a,b). Esseacúmulo decorreda aplicação freqüente de fertilizantes em pequenas profundidades e é devido à contribuição da fixação biológica de $\mathrm{N}$ das leguminosas, aliada à deposição de resíduos de culturas sobre a superfície, os quais aí permanecem pel o não-revolvimento do solo.

Este trabal ho teve como objetivo avaliar o efeito de sistemas de produção de grãos e de pastagens anuais de inverno e pastagens perenes, sob PD, após cinco anos de cultivo, sobre o nível de fertilidade do solo, no Planal to Médio do RS.

\section{MATERIAL E MÉTODOS}

O estudo foi executado num experimento realizado na E mbrapa-Centro Nacional de Pesquisa deTrigo (E mbrapa Trigo), município de Passo F undo (RS) $\left(28^{\circ} 15^{\prime} \mathrm{S}, 52^{\circ} 24^{\prime} \mathrm{W}\right.$ de latitude e $684 \mathrm{~m}$ de altitude), no período de 1993 a 1997, em Latossolo Vermel ho distrófico típico (EMBRAPA, 1999) textura muito argilosa relevo suave ondulado. Os teores médi os de argila, silte e areia são, respectivamente: 72,13 e $15 \%$. Nesse mesmolocal, antes da instalação do experimento, foram plantadas lavouras de cevada ou de trigo, no inverno, e de soja, no verão.

Os tratamentos consistiram de quatro sistemas de produção integrando produção de grãos [aveia branca (Avena sativa L.), milho (Zea mays L.), soja (Glycinemax Merril) e trigo (Triticum aestivumL)], 
pastagens anuais de inverno [aveia preta (Avena strigosa Schred.), azevém (Lolium multiflorum L.) e ervilhaca (Vicia sativa L.)]e pastagens perenes [alfafa (Medicago sativa L), cornichão (Lotus corniculatus L.), festuca (Festuca arundinacea Schred.), pensacola (Paspalum notatum Flügge), trevo branco (Trifolium repens L.) e trevo vermelho (Trifolium pratenseL.)]- sistema I (trigo/soja, aveia branca/soja eervilhaca/milho); sistema II (trigo/soja, aveia branca/soja e aveia preta + ervilhaca pastejadas/milho); sistema III [pastagens perenes da estação fria (festuca + trevo branco + cornichão)]; sistema IV [pastagens perenes da estação quente (pensacola +aveia preta +azevém + trevovermelho + cornichão)]; e sistema $\mathrm{V}$ (alfafa para feno, como tratamento adicional com repetições em áreas contíguas ao experimento, estabel ecido em 1994). As culturas produtoras de grãos, tanto no inverno como no verão, bem como as pastagens anuais de inverno, foram estabel ecidas sob sistema plantio direto. As pastagens perenes de estação fria e de estação quente foram estabel ecidas associadas com trigo em 1993. As pastagens anuais de inverno eas pastagens perenes foram pastejadas por animais mistos, duas e cinco vezes por ano, respectivamente.

E mabril de1993, antes da semeadura das culturas deinverno, foram col etadas amostras de solo em cada parcela, a uma profundi dade de $0-20 \mathrm{~cm}$, cujos valores médios foram: $\mathrm{pH}=6,0 ; \mathrm{Al}$ trocável $=0,50 \mathrm{mmol}_{\mathrm{C}} \mathrm{dm}^{-3}$; $\mathrm{Ca}+\mathrm{Mg}$ trocáveis $=102,8 \mathrm{mmol}_{\mathrm{c}} \mathrm{dm}^{-3}$; matéria orgânica = 23,0 $\mathrm{g} \mathrm{kg}^{-1}$; P extraível $=5,3 \mathrm{mg} \mathrm{kg}^{-1}$; e $\mathrm{K}$ trocável $=60 \mathrm{mg} \mathrm{kg}^{-1}$. Três anos da instalação do experimento, foi efetuada calagem com cal cário dolomítico, com base no método SMP $(\mathrm{pH} \mathrm{6,0).} \mathrm{As}$ parcelas semeadas com alfafa foram corrigidas novamente com 6,0 t de calcário por hectare (PRNT 100\%), para elevar o pH para 6,5, aplicadas em duas vezes: metade antes da aração (arado de discos) emetade antes da gradagem (gradedediscos).

No sistema I e no II, a pastagem de aveia preta + ervilhaca, a aveia branca, o milho e o trigo, além da adubação de manutenção, receberam também adubação nitrogenada de cobertura de acordo com a recomendação.

Em maio de 1998, após a col heita das culturas de verão, foram coletadas amostras de solo compostas de quatro subamostras por parcela, em cada uma das seguintes profundidades: 0-5, 5-10, 10-15 e 15$20 \mathrm{~cm}$. As análises ( $\mathrm{pH}$ em água, $\mathrm{P}$ extraível, $\mathrm{K}$ trocável, matéria orgânica, Al trocável e $\mathrm{Ca}+\mathrm{Mg}$ trocáveis) seguiram o método descrito por Tedesco et al. (1985): Al (extração em solução de KCL 1 mol L-1 e determinado por titulometria com solução $\mathrm{NaOH}$ 0,025); $\mathrm{Ca}+\mathrm{Mg}$ (mesmo extrator doAl edeterminado por espectrofotometria deabsorção atômica); matéria orgânica (determinada por combustão úmida), eP e $\mathrm{K}$ (determinados por Mehlich-1). O delineamento experimental foi em blocos ao acaso, com quatro repetições. A área de cada parcela foi de $20 \mathrm{~m}$ de comprimento por $20 \mathrm{~m}$ de largura $\left(400 \mathrm{~m}^{2}\right)$.
Os diversos sistemas de produção, integrando pastagens anuais de inverno com produção de grãos e pastagens perenes, foram comparados para cada característica de fertilidade de solo em determinada profundidade de amostragem. As profundidades de amostragem de solo foram comparadas dentro de um mesmo sistema de produção. Todas as comparações foram efetuadas por meio de contrastes com um grau de liberdade (Steel \& Torrie, 1980). A significância dos contrastes foi dada pelo teste $F$, levando em conta o desdobramento dos graus de liberdade do erro.

\section{RESULTADOS E DISCUSSÃO}

$\mathrm{O}$ pH do solo, após cinco anos de cultivo sob sistema plantio direto (PD), na camada de $0-5 \mathrm{~cm}$, nos quatro primeiros sistemas, nos quais não se aplicou calcário (Quadro 1), apresentou valores menores do que o valor verificado na camada de 0-20 cm de profundidade, por ocasião da instalação do experimento. A perda gradual do efeito residual da calagem que fora realizada antes do início deste estudo explica as alterações observadas. Resultados semel hantes foram observados por Santos \& Tomm (1996a) em sistemas de rotação de culturas para trigo, sob PD, no Paraná.

Parte da resposta positiva das culturas à calagem pode ocorrer pelo aumento de absorção de $\mathrm{N}$ pelas plantas (Edmeades et al., 1981). A acidificação do solo tende a reduzir a absorção de N, que, por sua vez, limita o crescimento de plantas. Isso não foi verificado entre os sistemas de produção estudados, devi do ao acúmulo de matéria orgânica na superfície em função do PD. Nos quatro primeiros sistemas, houve acidificação da camada de $0-5 \mathrm{~cm}$, necessitando nova calagem após cinco anos para o cultivo eficiente de leguminosas (SBCS, 1995).

No sistema $\mathrm{V}$, o maior valor de $\mathrm{pH}$ na camada de 0-5 cm observado após este período de estudo está relacionado com a aplicação de 6,0 t de cal cário por hectare (PRNT 100\%), em abril de 1994. Resultados opostos foram obtidos por outros autores. No trabal ho realizado por Shear \& M oschler (1969), nos Estados Unidos da América, em Podzólico BrunoAcinzentado, em que aplicou 1,1 t de calcário por hectare, três anos antes da primeira avaliação, os resultados foram diferentes, ou seja, o valor de $\mathrm{pH}$ na camada de $0-5 \mathrm{~cm}$ foi menor, em comparação aos valores de $\mathrm{pH}$ na camada de $0-20 \mathrm{~cm}$, antes do estabel ecimento do experimento. No Paraná, Sidiras \& Pavan (1985), após quatro anos sob PD, igualmente verificaram valores de $\mathrm{pH}$ em Latossolo Roxo distrófico, na camada de $0-10 \mathrm{~cm}$ menores do que aqueles registrados antes da instalação do experimento na camada de $0-20 \mathrm{~cm}$. A aplicação de calcário no sistema $\vee$ foi mais que suficiente para manter o pH em nível recomendado (SBCS, 1995). 
Quadro 1. Valores médios de pH em água, de alumínio trocável e de cálcio + magnésio trocáveis, avaliados após as culturas de verão de 1998, em quatro camadas de solo e para diferentes sistemas de rotação de culturas

\begin{tabular}{|c|c|c|c|c|c|c|c|c|c|c|}
\hline \multirow{2}{*}{$\begin{array}{l}\text { Sistema de } \\
\text { rotação(1) }\end{array}$} & \multicolumn{10}{|c|}{ Profundidade $(\mathrm{cm})$} \\
\hline & $0-5$ & $5-10$ & 10-15 & $15-20$ & $0-5 \times 5-10$ & $0-5 \times 10-15$ & $0-5 \times 15-20$ & $5-10 \times 10-15$ & $5-10 \times 15-20$ & $10-15 \times 15-$ \\
\hline \multicolumn{5}{|c|}{$\ldots$ pH (água $1: 1$ ) } & \multicolumn{6}{|c|}{ - Contrastes entre profundidades $(P>F)$} \\
\hline 1 & 5,87 & 6,13 & 6,34 & 6,16 & $* *$ & $* *$ & $* *$ & $*$ & ns & ns \\
\hline II & 5,95 & 6,19 & 6,37 & 6,14 & $*$ & $* *$ & ns & ns & ns & $*$ \\
\hline III & 5,83 & 6,32 & 6,60 & 6,20 & ns & $* *$ & ns & ns & ns & ns \\
\hline IV & 5,83 & 6,30 & 6,50 & 6,47 & $* *$ & $* *$ & $* *$ & $*$ & ns & ns \\
\hline $\mathrm{V}$ & 6,30 & 6,50 & 6,57 & 6,37 & ns & ns & ns & ns & ns & ns \\
\hline \multicolumn{11}{|c|}{ Contrastes entre sistemas } \\
\hline$|\times| \mid$ & ns & ns & ns & ns & & & & & & \\
\hline$|\times|||$ & ns & ns & ns & ns & & & & & & \\
\hline $\mathrm{I} \times \mathrm{IV}$ & ns & ns & ns & ns & & & & & & \\
\hline $\mathrm{I} \times \mathrm{V}$ & $*$ & $*$ & ns & ns & & & & & & \\
\hline $11 \times 111$ & ns & ns & ns & ns & & & & & & \\
\hline II $\times$ IV & ns & ns & ns & $*$ & & & & & & \\
\hline II $x \mathrm{~V}$ & $*$ & ns & ns & ns & & & & & & \\
\hline $\mathrm{III} \times \mathrm{IV}$ & ns & ns & ns & ns & & & & & & \\
\hline III $x \mathrm{~V}$ & $*$ & ns & ns & ns & & & & & & \\
\hline $\mathrm{IV} \times \mathrm{V}$ & $*$ & ns & ns & ns & & & & & & \\
\hline \multicolumn{5}{|c|}{ Al $\left(\mathrm{mmol}_{\mathrm{c}} \mathrm{dm}^{-3}\right)$} & \multicolumn{6}{|c|}{ - Contrastes entre profundidades $(P>F)$} \\
\hline 1 & 0,38 & 0,04 & 0,00 & 0,13 & $*$ & $*$ & ns & ns & ns & ns \\
\hline II & 0,54 & 0,13 & 0,00 & 0,17 & $*$ & $* *$ & $*$ & ns & ns & ns \\
\hline 111 & 0,63 & 0,13 & 0,00 & 0,38 & ns & ns & ns & ns & ns & ns \\
\hline IV & 0,50 & 0,13 & 0,00 & 0,00 & ns & $*$ & $*$ & ns & ns & ns \\
\hline V & 0,13 & 0,00 & 0,00 & 0,00 & ns & ns & ns & ns & ns & ns \\
\hline
\end{tabular}

Contrastes entre sistemas

\begin{tabular}{|c|c|c|c|}
\hline $1 \times 1 \mid$ & ns & ns & ns \\
\hline $1 \times 1||$ & ns & ns & ns \\
\hline $1 \times 1 V$ & ns & ns & ns \\
\hline $\mathrm{I} \times \mathrm{V}$ & ns & ns & ns \\
\hline $11 \times 111$ & ns & ns & ns \\
\hline II $\times 1 \mathrm{~V}$ & ns & ns & ns \\
\hline$I I \times V$ & ns & ns & ns \\
\hline III $\times 1 \mathrm{~V}$ & ns & ns & ns \\
\hline III $x \mathrm{~V}$ & ns & ns & ns \\
\hline IV $\times V$ & ns & ns & ns \\
\hline
\end{tabular}

\begin{tabular}{|c|c|c|c|c|c|c|c|c|c|c|}
\hline & $\mathrm{Ca}+$ & $(\mathrm{mm}$ & $\left(\mathrm{dm}^{-3}\right)$ & - & & & 5 en & und & $>$ & \\
\hline 1 & 84 & 89 & 95 & 90 & ns & ns & ns & $*$ & ns & ns \\
\hline II & 86 & 87 & 93 & 87 & ns & $*$ & ns & ns & ns & ns \\
\hline III & 89 & 100 & 101 & 98 & ns & ns & ns & ns & ns & ns \\
\hline IV & 95 & 98 & 96 & 96 & ns & ns & ns & ns & ns & ns \\
\hline V & 105 & 103 & 108 & 99 & ns & ns & ns & ns & ns & ns \\
\hline
\end{tabular}

\begin{tabular}{lcccc}
\multicolumn{5}{c}{ Contrastes entre sistemas } \\
\hline $\mathrm{I} \times \mathrm{II}$ & $\mathrm{ns}$ & $\mathrm{ns}$ & $\mathrm{ns}$ & $\mathrm{ns}$ \\
$\mathrm{I} \times \mathrm{III}$ & $\mathrm{ns}$ & $\mathrm{ns}$ & $\mathrm{ns}$ & $\mathrm{ns}$ \\
$\mathrm{I} \times \mathrm{IV}$ & $*$ & $\mathrm{~ns}$ & $\mathrm{~ns}$ & $\mathrm{~ns}$ \\
$\mathrm{I} \times \mathrm{V}$ & $* *$ & $*$ & $*$ & $\mathrm{~ns}$ \\
$\mathrm{II} \times \mathrm{III}$ & $\mathrm{ns}$ & $*$ & $\mathrm{~ns}$ & $*$ \\
$\mathrm{II} \times \mathrm{IV}$ & $\mathrm{ns}$ & $\mathrm{ns}$ & $\mathrm{ns}$ & $\mathrm{ns}$ \\
$\mathrm{II} \times \mathrm{V}$ & $* *$ & $*$ & $* *$ & $*$ \\
$\mathrm{III} \times \mathrm{IV}$ & $\mathrm{ns}$ & $\mathrm{ns}$ & $\mathrm{ns}$ & $\mathrm{ns}$ \\
$\mathrm{III} \times \mathrm{V}$ & $*$ & $\mathrm{~ns}$ & $\mathrm{~ns}$ & $\mathrm{~ns}$ \\
$\mathrm{IV} \times \mathrm{V}$ & $\mathrm{ns}$ & $\mathrm{ns}$ & $\mathrm{ns}$ & $\mathrm{ns}$
\end{tabular}

(1) I: trigo/soja, aveia branca/soja e ervilhaca/milho; II: trigo/soja, aveia branca/soja pastagem de aveia preta + ervilhaca/milho; III : pastagem perene de inverno; IV pastagem perene de verão; e V alfafa.

*, ** e ns: Significativos a 1 e 5\% e não-significativo, respectivamente. 
O sistema $\mathrm{V}$ apresentou valor significativamente maior de $\mathrm{pH}$ na camada de $0-5 \mathrm{~cm}$ do que o dos demais sistemas (Quadro 1). Esseresultado repetiuse na camada de $5-10 \mathrm{~cm}$ quando o $\mathrm{pH}$ do sistema $\mathrm{V}$ foi superior ao do sistema I. I sso pode ser atribuído à aplicação de calcário, em 1994, no sistema V. O sistema IV manifestou maior valor depH na camada de 15-20 cm, em relação ao sistema II.

Em alguns sistemas de produção, foram observadas diferenças significativas do val or de $\mathrm{pH}$ do solo entre determinadas profundidades de amostragem. Todavia, o sistema $V$ não diferiu entre as profundidades de amostragem para os val ores de $\mathrm{pH}$. Demaneira geral, os valores de $\mathrm{pH}$ aumentaram gradativamente com o aumento da profundidade do solo (0-5 cm e 10-15 cm). Resultados semel hantes foram obtidos por Santos \& Tomm (1996a), trabalhando com sistemas de rotação de culturas para trigo, sob PD, no Paraná. Sidiras \& Pavan (1985) obtiveram resultado contrário, isto é, valor decrescente de $\mathrm{pH}$ na camada de $0-10 \mathrm{~cm}$, em relação à camada de $0-20 \mathrm{~cm}$, também no Paraná. De 15 a $20 \mathrm{~cm}$, houve decréscimo nos valores de pH em todos os sistemas de produção. O acúmulo de palha na superfície acidifica o solo (Haynes, 1986).

O valor de Al trocável do solo (Quadro 1), em todas as profundidades, nos sistemas IV eV, foi igual ou inferior ao registrado por ocasião da instalação do experimento. A diminuição do teor deAl trocável no sistema $V$ deveu-se à quantidade de calcário aplicada em abril de 1994.

Não foram observadas diferenças significativas entre teor de Al trocável dos cincos sistemas de produção estudados. Dados semelhantes foram encontrados por Santos \& Tomm (1996b; 1998), trabalhando com sistemas de rotação de culturas para triticale, após seis anos de cultivo, sob PD, em Latossolo Vermel ho distrófico típico, no Rio Grande do Sul, e com sistemas de rotação de culturas para cevada, após dez anos de cultivo, sob PD, em Latossolo Bruno Alumínico câmbico, no Paraná.

Em três dos cincos sistemas de produção, foram verificadas diferenças significativas no nível de Al trocável entre as profundidades de amostragem do solo. Contudo, os sistemas III e V não diferiram entre as profundidades de amostragem para o valor de Al trocável. Nos sistemas I, II e IV, o valor de Al trocável do solo diminuiu da camada de $0-5 \mathrm{~cm}$ para a camada de $10-15 \mathrm{~cm}$. Dados similares foram observados por Santos \& Tomm (1996a), trabalhando com sistemas de rotação de culturas para trigo, sob PD, no Paraná. Sidiras \& Pavan (1985) obtiveram resultados opostos, ou seja, valor menor de Al trocável, na camada de $0-10 \mathrm{~cm}$, igualmente no Paraná.

Neste período de estudo e nos quatro primeiros sistemas de produção, observaram-se diminuição do valor de $\mathrm{pH}$ e aumento no teor de Al trocável na camada de 0-5 cm, em relação ao valor em 1993, caracterizando uma acidificação nos primeiros centímetros do solo. I sso pode ser atribuído à aplicação de fertilizantes nitrogenados, nos dois primeiros sistemas de produção, e à mineralização de resíduos culturais na superfície do solo (Salet, 1994). Para os demais sistemas, essa explicação não é totalmente válida, já que não foi aplicado fertilizante nitrogenado e grande parte da massa vegetal foi consumida pelos animais.

O valor médio de $\mathrm{Ca}+\mathrm{Mg}$ trocáveis do solo (Quadro 1), em todas as camadas, é consi derado alto para o crescimento e desenvolvimento de culturas na região (SBCS, 1995). A área experimental havia sido corrigida com cal cário dol omítico anos antes do início do referido experimento. A aplicação de calcário dolomítico, em que tanto o cálcio como o magnési o foram fornecidos em grandes quantidades, fez com que os respectivos teores ultrapassassem os níveis críticos exigidos pelas espécies que compuseram os sistemas de rotação (SBCS, 1995). Todavia, esses teores encontraram-se abaixo do val or medido antes do início do experimento nos quatro primeiros sistemas de produção, enquanto, no sistema $V$, esses valores mantiveram-se acima nas camadas de $0-5 \mathrm{~cm}$ a $10-15 \mathrm{~cm}$. Nos trabalhos efetuados por Santos \& Tomm (1996a), os val ores de $\mathrm{Ca}+\mathrm{Mg}$ trocáveis foram superiores àqueles observados no início do experimento.

Em alguns sistemas de produção, houve diferenças significativas quanto aos teores de $\mathrm{Ca}+\mathrm{Mg}$ trocáveis, dentro da mesma profundidade de amostragem. O sistema $\mathrm{V}$ apresentou valores mai ores de $\mathrm{Ca}+\mathrm{Mg}$ trocáveis na camada de 0-5 cm do que os dos sistemas II ell I I sso pode ser devido à aplicação de calcário, em 1994, no sistema V. Da mesma forma e na mesma camada de solo, o sistema IV foi superior ao sistema I. A superioridade do sistema $V$ repetiu-se na camada de $5-10 \mathrm{~cm}$, em comparação aos sistemas I e II. Além disso, o sistema III foi superior ao sistema II para os valores de $\mathrm{Ca}+\mathrm{Mg}$ trocáveis do solo. O sistema $\mathrm{V}$ foi ainda superior aos sistemas I e II na camada de $10-15 \mathrm{~cm}$ e somente ao sistema II na camada de $15-20 \mathrm{~cm}$. Nesta última camada, o sistema III apresentou maiores teores de $\mathrm{Ca}+\mathrm{Mg}$ trocáveis do que o sistema II. Santos \& Tomm (1996a), no estado do Paraná, não observaram diferenças significativas entre os sistemas de rotação de culturas para trigo.

Nas comparações dentro de um mesmo sistema de rotação, foram observadas diferenças significativas de $\mathrm{Ca}+\mathrm{Mg}$ trocáveis do solo entre determinadas profundidades de amostragem, em alguns tratamentos. Nos sistemas I e II, os teores de $\mathrm{Ca}+\mathrm{Mg}$ trocáveis do solo aumentaram da camada de 5-10 cm para $10-15 \mathrm{~cm}$ ou da camada de 0-5 cm para $10-15 \mathrm{~cm}$, respectivamente. Resultado similar foi encontrado por Santos \& Tomm (1996a) em sistemas de rotação de culturas para trigo, após 
4,5 anos, sob PD, em Latossolo Bruno Alumínico câmbico, no Paraná. Por outrolado, Sidiras \& Pavan (1985), também no Paraná, observaram maiores valores de $\mathrm{Ca}+\mathrm{Mg}$ trocáveis na camada superficial $(0-10 \mathrm{~cm})$, em relação à mais profunda $(10-20 \mathrm{~cm})$. Essa explicação também é respaldada pelos resultados de $\mathrm{pH}$ e de Al trocável. Os sistemas III, IV e V não diferiram entre as profundidades de amostragem para os valores de Ca + Mg trocáveis. De 15 a 20 cm, em alguns sistemas de produção, houve decréscimo nos val ores de Ca + Mg trocáveis.

Os valores de matéria orgânica do solo, observados em todas as camadas (Quadro 2), mantiveram-se próximos, iguais ou superiores ao valor medido na camada de $0-20 \mathrm{~cm}$, por ocasião da instalação do experimento, indicando que a adoção do PD pode contribuir para aumento do nível de matéria orgânica e, conseqüentemente, da fertilidade do solo, independentemente da quantidade de fertilizante aplicado. N os primeiros anos de adoção do PD, existe tendência à elevação do valor de matéria orgânica nas camadas próximas à superfície do solo, pois o nível de equílíbrio situa-se em valores intermediários entre aquel es sob vegetação natural e aqueles sob cultivo convencional. Dessa forma, o sistema plantio direto apresenta maior valor de matéria orgânica e de maior força iônica da solução de solo na camada superficial (Salet, 1994). I sso explica, em parte, a não-ocorrência de toxidez de alumínio, quando menor atividade iônica do alumínio.

Nos sistemas de produção estudados, em al gumas camadas de amostragem, houve diferenças significativas entreas médias para matéria orgânica do solo. O sistema IV mostrou teor de matéria orgânica maior do que o dos sistemas I e II, na camada de $0-5 \mathrm{~cm}$. Nessa mesma camada, os sistemas III e V foram superiores ao II, para os valores de matéria orgânica. Essa diferença entre os sistemas pode ser explicada, em parte, pela presença de leguminosas perenes para pastoreio ou corte (alfafa, cornichão e trevo vermelho), nos sistemas IV eV, em comparaçãoaos sistemas I (trigo/ soja, aveia branca/soja e ervilhaca/milho) ell (trigo/ soja, aveia branca/soja e pastagem de aveia preta +ervilhaca/milho). O uso de leguminosas para reciclagem de nutrientes e aumento do teor de $\mathrm{N}$ dos sistemas pode ser uma estratégia para atingir uma agricultura sustentável. Na camada de 10$15 \mathrm{~cm}$, o sistema $V$ foi superior aos sistemas I , II e IV, e o sistema III ao II, para o nível de matéria orgânica. Além disso, o sistema III foi superior aos sistemas I e II , na camada de $15-20 \mathrm{~cm}$. Resultados semelhantes foram obtidos por Santos \& Tomm (1996a), trabalhando com sistemas de produção de grãos envolvendo trigo e outras culturas de inverno e de verão, sob PD, no Paraná.

Foram verificadas diferenças significativas no teor de matéria orgânica entre determinadas profundidades de amostragem do solo na maioria dos sistemas de produção. O sistema I, porém, não diferiu entre as profundidades de amostragem para os val ores de matéria orgânica. Os valores de matéria orgânica do solo decresceram progressivamente da camada de $0-5 \mathrm{~cm}$ para a camada de $10-15 \mathrm{~cm}$. Tendência semel hante nas variações entre nível de matéria orgânica da camada de $0-5 \mathrm{~cm}$ para a camada de 15-20 cm foi verificada por Sá (1993), por Santos \& Tomm (1996a), em sistemas de rotação de culturas para trigo, e por Santos \& Tomm (1996b), em sistemas de rotação de culturas para triticale, estes últimos sob PD. A manutenção do nível de matéria orgânica em valores mais el evados apenas na camada superficial do solo decorre do acúmulo de resíduos vegetais sobre a superfície do solo sob PD, pela ausência de incorporação física destes ao solo, o que diminui a taxa demineralização. Resultados semel hantes foram obtidos por Muzilli (1983).

O teor deP extraível dosolo, na primeira camada de solo $(0-5 \mathrm{~cm})$, esteve acima do valor considerado crítico, nesse tipo de solo, para o crescimento e desenvolvimento das culturas (REUNIÃO..., RCSBPT, 1999) (Quadro 2). Também na primeira camada de solo, o teor de P extraível foi mais el evado do que o teor verificado antes do início do experimento, na camada de 0-20 cm. O PD provoca alterações nas propriedades químicas do solo, as quais, por sua vez, refletem-se na fertilidade e na eficiência do uso de nutrientes pelas espécies. Além disso, a rotação de culturas ou sistemas de produção têm importante papel na reciclagem de nutrientes, uma vez que, deacordo com Mengel \& Kirkby (1987), as espécies vegetais diferem entre si com referência à quantidade de resíduos fornecidos, à eficiência de absorção de íons e à exploração de diferentes profundidades de solo.

$\mathrm{Na}$ camada de $5-10 \mathrm{~cm}$, houve diferenças significativas entre os sistemas de produção para o valor do P extraível do solo. O sistema II foi superior aos sistemas III e V. As demais camadas avaliadas não diferiram entre os sistemas de produção estudados.

Todos os sistemas avaliados diferiram significativamente quanto ao val or deP extraível na maioria das profundidades de amostragem. Em todos os sistemas, o teor de $\mathrm{P}$ extraível na camada de $0-5 \mathrm{~cm}$ foi de 2,5 a 5,4 vezes superior ao verificado na camada de $15-$ $20 \mathrm{~cm}$. Resultados similares foram observados em outros estudos, sob PD, por Shear \& Moschler (1969), por Triplett J r. \& Van Doren J r. (1969), por Sá (1993) e por Santos \& Tomm (1996a). Segundo Sidiras \& Pavan (1985), o acúmulo de P extraível próximo à superfície do solo decorre das aplicações anuais de fertilizantes fosfatados, da liberação de P durante a decomposição de resíduos vegetais e da menor fixação de $\mathrm{P}$, proveniente do menor contato desse elemento com os constituintes inorgânicos do solo, uma vez que não há revolvimento de solo no PD. Em parte, essas afirmações também são válidas para o K trocável do solo. 
Quadro 2. Teores médios de matéria orgânica, de fósforo extraível e de potássio trocável, avaliados após as culturas de verão de 1998, em quatro camadas de solo e para diferentes sistemas de rotação de culturas

\begin{tabular}{|c|c|c|c|c|c|c|c|c|c|c|}
\hline \multirow{2}{*}{$\begin{array}{l}\text { Sistema de } \\
\text { rotação(1) }^{(1)}\end{array}$} & \multicolumn{10}{|c|}{ Profundidade $(\mathrm{cm})$} \\
\hline & 0-5 & $5-10$ & 10-15 & $15-20$ & $0-5 \times 5-10$ & $0-5 \times 10-15$ & $0-5 \times 15-20$ & $5-10 \times 10-15$ & $5-10 \times 15-20$ & $10-15 \times 15-2 C$ \\
\hline \multicolumn{5}{|c|}{ _ Matéria orgânica $\left(\mathrm{g} \mathrm{kg}^{-1}\right)$} & \multicolumn{6}{|c|}{ - Contrastes entre profundidades $(\mathrm{P}>\mathrm{F})$} \\
\hline 1 & 30 & 25 & 23 & 23 & ns & ns & ns & ns & ns & ns \\
\hline II & 28 & 24 & 22 & 23 & $* *$ & $* *$ & $* *$ & ns & ns & ns \\
\hline IV & 37 & 25 & 23 & 23 & $* *$ & $* *$ & $* *$ & ns & ns & ns \\
\hline $\mathrm{V}$ & 32 & 25 & 25 & 24 & $* *$ & $* *$ & $* *$ & ns & ns & ns \\
\hline
\end{tabular}

Contrastes entre sistemas

\begin{tabular}{|c|c|c|c|c|}
\hline $1 \times 1 \mid$ & ns & ns & ns & ns \\
\hline $1 \times 111$ & ns & ns & ns & * \\
\hline $1 \times 1 \mathrm{~V}$ & $* *$ & ns & ns & ns \\
\hline $\mathrm{I} \times \mathrm{V}$ & ns & ns & $* *$ & ns \\
\hline $11 \times 111$ & $*$ & ns & * & * \\
\hline II $\times$ IV & $* *$ & ns & ns & ns \\
\hline $\mathrm{II} \times \mathrm{V}$ & * & ns & $* *$ & ns \\
\hline III x IV & ns & ns & ns & ns \\
\hline III $\times \mathrm{V}$ & ns & ns & ns & ns \\
\hline IV $\times V$ & ns & ns & * & ns \\
\hline \multicolumn{5}{|c|}{$-P\left(\mathrm{mg} \mathrm{kg}^{-1}\right)$} \\
\hline 1 & 17,5 & 10,0 & 6,8 & 5,8 \\
\hline II & 14,9 & 13,1 & 7,8 & 5, \\
\hline III & 11,0 & 4,3 & 2,9 & 3,0 \\
\hline IV & 14,4 & 7,8 & 4,9 & 2,7 \\
\hline V & 18,6 & 5,2 & 3,9 & 3,2 \\
\hline
\end{tabular}

\begin{tabular}{lccccc}
\multicolumn{7}{c}{ Contrastes entre profundidades $(\mathrm{P}>\mathrm{F})$} & \\
\hline$* *$ & $* *$ & $* *$ & $\mathrm{~ns}$ & $*$ & $\mathrm{~ns}$ \\
$\mathrm{~ns}$ & $*$ & $* *$ & $\mathrm{~ns}$ & $*$ & $\mathrm{~ns}$ \\
$* *$ & $* *$ & $* *$ & $\mathrm{~ns}$ & $\mathrm{~ns}$ & $\mathrm{~ns}$ \\
$* *$ & $* *$ & $* *$ & $\mathrm{~ns}$ & $*$ & $\mathrm{~ns}$ \\
$* *$ & $* *$ & $* *$ & $\mathrm{~ns}$ & $\mathrm{~ns}$ & $\mathrm{~ns}$
\end{tabular}

Contrastes entre sistemas

$\begin{array}{lcccc}I \times I I & n s & n s & n s & n s \\ I \times I I I & n s & n s & n s & n s \\ I \times I V & n s & n s & n s & n s \\ I \times V & n s & n s & n s & n s \\ I I \times I I I & n s & * & n s & n s \\ I I \times I V & n s & n s & n s & n s \\ I I \times V & n s & * & n s & n s \\ I I I \times I V & n s & n s & n s & n s \\ I I I \times V & n s & n s & n s & n s \\ I V \times V & n s & n s & n s & n s\end{array}$

\begin{tabular}{|c|c|c|c|c|}
\hline I & 142 & 84 & 59 & 52 \\
\hline II & 97 & 68 & 52 & 49 \\
\hline 111 & 58 & 23 & 15 & 14 \\
\hline IV & 82 & 50 & 45 & 35 \\
\hline $\mathrm{V}$ & 150 & 102 & 63 & 46 \\
\hline
\end{tabular}

\begin{tabular}{llllll}
\multicolumn{7}{c}{ Contrastes entre profundidades $(\mathrm{P}>\mathrm{F})$} \\
& $* *$ & $* *$ & $\mathrm{~ns}$ & $\mathrm{~ns}$ & $\mathrm{~ns}$ \\
$\mathrm{~ns}$ & $* *$ & $* *$ & $\mathrm{~ns}$ & $\mathrm{~ns}$ & $\mathrm{~ns}$ \\
$* *$ & $* *$ & $* *$ & $\mathrm{~ns}$ & $\mathrm{~ns}$ & $\mathrm{~ns}$ \\
$* *$ & $* *$ & $* *$ & $\mathrm{~ns}$ & $\mathrm{~ns}$ & $\mathrm{~ns}$ \\
$* *$ & $* *$ & $* *$ & $\mathrm{~ns}$ & $\mathrm{~ns}$ & $\mathrm{~ns}$
\end{tabular}

Contrastes entre sistemas

$\begin{array}{lcccc}\mathrm{I} \times \mathrm{II} & * * & \mathrm{~ns} & \mathrm{~ns} & \mathrm{~ns} \\ \mathrm{I} \times \mathrm{III} & * * & * * & * * & * * \\ \mathrm{I} \times \mathrm{IV} & * * & * & \mathrm{~ns} & \mathrm{~ns} \\ \mathrm{I} \times \mathrm{n} & \mathrm{ns} & \mathrm{ns} & \mathrm{ns} & \mathrm{ns} \\ \mathrm{II} \times \mathrm{III} & \mathrm{ns} & * * & * * & * \\ \mathrm{II} \times \mathrm{IV} & \mathrm{ns} & \mathrm{ns} & \mathrm{ns} & \mathrm{ns} \\ \mathrm{II} \times \mathrm{V} & * & * & \mathrm{~ns} & \mathrm{~ns} \\ \mathrm{III} \times \mathrm{IV} & \mathrm{ns} & \mathrm{ns} & * & \mathrm{~ns} \\ \mathrm{III} \times \mathrm{V} & * * & * * & * * & \mathrm{~ns} \\ \mathrm{IV} \times \mathrm{V} & * & * * & \mathrm{~ns} & \mathrm{~ns}\end{array}$

(1) I: trigo/soja, aveia branca/soja e ervilhaca/milho; Il: trigo/soja, aveia branca/soja pastagem de aveia preta + ervilhaca/milho; III : pastagem perene de inverno; IV pastagem perene de verão; e V alfafa.

*, ** e ns: Significativos a 1 e $5 \%$ e não-significativo, respectivamente. 
Após cinco anos de PD, somente o teor de K trocável, na camada de 0-5 cm (Quadro 2), nos sistemas I, II, IV e V, e na camada $5-10 \mathrm{~cm}$, nos sistemas I e V, foi significativamente superior ao valor considerado crítico para o crescimento e desenvolvimento das culturas ( $80 \mathrm{mg} \mathrm{kg}^{-1}$ ) (RCSBPT, 1999). Além disso, o teor deK trocável observado nas camadas esistemas de produção supracitados, mais o sistema II, esteve próximo ou acima do valor registrado antes do início deste experimento.

Neste período de estudo, os teores de K trocável do solo diferiram significativamente entre alguns sistemas produção. Os sistemas I eV, na camada de 0-5 cm, foram superiores aos sistemas II, III e IV para o teor de K trocável. Por sua vez, o sistema I, na camada de $5-10 \mathrm{~cm}$, foi superior aos sistemas III e IV. Além disso, o sistema $V$ foi superior ao sistema II. Nessa mesma camada e nas camadas de 10-15 cm e 15-20 cm desolo, osistema II foi superior ao sistema III. Os sistemas I e V, na camada de 10-15 cm, foram superiores ao sistema III. Por sua vez, o sistema IV também foi superior ao sistema III, nessa mesma camada. Na camada de $15-20 \mathrm{~cm}$, o sistema I foi superior ao sistema III. Dados semelhantes foram obtidos por Santos \& Tomm $(1996 a, b)$, trabal hando com sistemas de rotação de culturas para trigo e para triticale, sob PD, no Paraná e no Rio Grande do Sul. Os valores mais elevados para o K trocável, observados nos sistemas I e V, deveram-se, provavel mente, à mai or quantidade de $\mathrm{K}$ aplicada nos referidos sistemas, em relação aos sistemas III e IV, e eventualmente não extraída e removida pelas culturas. No sistema II , foi aplicada a mesma quantidade de $\mathrm{K}$, porém parte desse elemento foi removida pelo pastejo da aveia preta +ervilhaca pelos animais.

Foram verificadas diferenças significativas de K trocável entre todas as profundidades de amostragem de sol o de todos os sistemas de produção avaliados. A exemplo do verificado com P extraível, também houve acúmulo de K trocável nas camadas próximas à superfície nos diferentes sistemas de rotação. O teor de K trocável decresceu com o aumento da profundidade de amostragem: na camada de $0-5 \mathrm{~cm}$ foi de 1,9 a 4,1 vezes maior que a concentração da camada de $15-20 \mathrm{~cm}$. Acúmulos semel hantes de K trocável, na camada de $0-5 \mathrm{~cm}$, em relação à camada de $15-20 \mathrm{~cm}$, em sistemas de rotação de culturas, sob PD, foram observados por Shear \& Moschler (1969), por Triplett J r. \& Van Doren J r. (1969), por Santos \& Tomm (1996a,b).

\section{CONCLUSÕES}

1. Os valores de $\mathrm{pH}$, de $\mathrm{Ca}+\mathrm{Mg}$ trocáveis, de matéria orgânica, de $\mathrm{P}$ extraível e de $\mathrm{K}$ trocável foram influenciados pel os sistemas de produção.
2. Os sistemas de produção sob plantio direto el evaram os teores de matéria orgânica, de P extraível e de $\mathrm{K}$ trocável, principalmente na camada de solo de $0-5 \mathrm{~cm}$, em relação ao preparo convencional de solo, para a mesma profundidade anteriormente à instalado do experimento.

3. Os sistemas com leguminosas perenes foram mais eficientes no acúmulo de matéria orgânica na camada superficial do solo.

4. Nos quatro primeiros sistemas, foram observados val ores menores de $\mathrm{pH}$ e de $\mathrm{Ca}+\mathrm{Mg}$ trocáveis na camada de $0-5 \mathrm{~cm}$, em relaçãoà de $15-20 \mathrm{~cm}$, enquanto com o teor de Al trocável ocorreu o contrário, indicando acidificação na camada superficial.

\section{LITERATURA CITADA}

CARPENEDO, V. \& MIELNICZUK, J. Estado de agregação e qualidade de agregados de Latossol os Roxos submetidos a diferentes sistemas de manejo. R. Bras. Ci. Solo, 14:99-105, 1990.

EDMEADES, D.C.; J UDO, M. \& SARATHCHANDRA, S.U. The effect of lime on nitrogen mineralization as measured by grass growth. Plant Soil, 60:177-186, 1981.

EMPRESA BRASILEIRA DE PESQUISA AGROPECUÁRIA EMBRAPA. Centro Nacional de Pesquisa de Solos, Rio de J aneiro. Sistema brasileiro de classificação de sol os. Rio de J aneiro, 1999. 412p.

HARRIS, R.F.; CHESTER, G. \& ALLEN, O.N. Dynamics of soil aggregation. Adv. Agron., 18:107-169, 1966.

HAYNES, R.J . The decomposition process: mineralization, humus formation, and degradation. In: HAYNES, R.J ., ed. Mineral nitrogen in the plant-soil system. Orlando, Academic Press, 1986. p.52-126. (Physiological Ecology. A Series of Monographs, Texts, and Treatises.)

MELLO, J S. Fundamentos para integração lavoura-pecuária no sistema plantio. R. Plantio Direto, 35:12-13, 1996.

MENGEL, K.\& KIRKBY, E.A. Principles of plant nutrition. 4.ed. Bern, International Potash Institute, 1987. 687p.

MUZI LLI, O. Influência do sistema de plantio direto, comparado ao convencional, sobre a fertilidade da camada arável do solo. R. Bras. Ci. Solo, 7:95-102, 1983.

REUNIÃO DA COMISSÃO SUL-BRASILEIRA DE PESQUISA DE TRIGO-RCSBPT, 31., 1999, PassoF undo. Recomendações da Comissão Sul-brasileira de Pesquisa de Trigo - 1999. Passo Fundo, 1999. 86p.

SÁ, J.C.M. Manejo da fertilidade do solo no sistema plantio direto. In: EMPRESA BRASILEIRA DE PESQUISA AGROPECUÁRIA. Centro Nacional de Pesquisa de Trigo (Passo Fundo, RS). Plantio direto no Brasil. Passo Fundo, 1993. p.37-60.

SALET, R.L Dinâmica de íons na solução de um solo submetido ao sistema plantio direto. Porto Alegre, Universidade Federal do Rio Grande do Sul, 1994. 111p. (Tese de Mestrado) 
SANTOS, H.P. \& TOMM, G.O. Estudos da fertilidade do solo sob quatro sistemas de rotação de culturas envolvendo trigo em plantio direto. R. Bras. Ci. Solo, 20:407-414, 1996a.

SANTOS, H.P. \& TOMM, G.O. Fertilidade do solo em rotação de culturas com triticale. R. Bras. Ci. Solo, 20:415-421, 1996b.

SANTOS, H.P. \& TOMM , G.O. Rotação de culturas para cevada, após dez anos: efeitos na fertilidade do solo. Ci. Rural, 28:573-580, 1998.

SHEAR, G.M. \& MOSCHLER, W.W. Continuous corn by the notillage and continuous tillage methods: a six-year comparison. Agron. J ., 58:524-526, 1969.

SIDIRAS, N. \& PAVAN, M.A. I nfluência do sistema de manejo do solo no seu nível de fertilidade. R. Bras. Ci.Solo, 9:249-254, 1985.
SOCIEDADE BRASILEIRA DE CIÊNCIA DO SOLO-SBCS/RSSC. Núdeo Regional Sul. Comissão de fertilidade do solo RS/SC. Recomendações de adubação e de calagem para os estados do Rio Grande do Sul e de Santa Catarina. 3.ed. Passo Fundo, 1995. 224p.

STEEL, G.D. \& TORRIE, J.H. Principles and procedures of statistics: a biometrical approach. 2.ed. New York, McGrawHill, 1980. 633p.

TEDESCO, M.J .; VOLKWEISS, S.J . \& BOHNEN, H. Análise de solos, plantas eoutros materiais. PortoAlegre, Universidade Federal do Rio Grande do Sul, 1985. 32p. (Boletim Técnico, 5)

TRIPLETT J r., G.B. \& van DOREN J r., D.M. Nitrogen, phosphorus, and potassium fertilization of non-tilled maize. Agron. J ., 61:637-639, 1969. 
H. P. SANTOS et al. 